\title{
TWO-STAGE TESTING TO ESTABLISH NON-INFERIORITY IN THE STRATIFIED $2 \times 2$ CONTINGENCY TABLES
}

\author{
Takashi Koshimizu* and Masaaki Tsujitani $^{\dagger}$
}

\begin{abstract}
In this article we propose a two-stage testing procedure for demonstrating noninferiority of a test drug in stratified $2 \times 2$ contingency tables, which includes a preliminary test of homogeneity of odds ratios. In order to prove that a new drug is not inferior to a control by more than a prescribed amount, we use model-based methods such as the weighted least-square (WLS) procedure by Grizzle et al. (1969), and test the null hypothesis of a specified non-unity ratio. Also included are some results of a Monte Carlo study conducted to investigate the accuracy of the proposed test to the null distribution, and power of the test under small and moderate sample size configurations.
\end{abstract}

\section{Introduction}

In some comparative clinical trials, a new drug is compared with an active control (standard drug). The usual test procedure verifies that a new drug has some advantages such as significant superiority to the standard drug in efficacy. If a new drug which offers ease of prescription, less adverse effects and so on, is shown to be as effective as a standard drug, then the new drug may be regarded as being preferable. It should, however, be noted that nonsignificance by a usual test of superiority never implies equivalence in a real sense, when the purpose of a clinical trial is to establish the equivalence that two treatments do not differ by more than a prescribed small amount(See, Yanagawa et al., 1992,1994).

In order to establish the equivalence of two treatments, several investigators have studied various statistical methods for testing the null hypothesis of non-zero difference or non-unity ratio in comparative binomial trials. Dunnett \& Gent (1977) introduced the hypothesis that the true difference is equal to a specified amount. Hirotsu(1986), Rodary et al.(1989) and Farrington \& Manning(1990) considered non-zero difference in a single $2 \times 2$ table. and the corresponding sample size formulae have been investigated (Makuch \& Simon, 1978; Blackwelder, 1982; Blackwelder \& Chang, 1984).

Alternatively, tests for non-unity relative risk or non-unity ratio have been proposed by Farrington \& Manning (1990) and Yanagawa et al.(1994). respectively. Farrington \& Manning (1990) applied the test statistic for the null hypothesis of non-zero difference or non-unity relative risk to studies of the pertussis vaccine, and argued that the problem was

\footnotetext{
* Graduate School of Engineering, Osaka Electro-Communication University, 18-8, Hatsu-cho, Neyagawa, Osaka 572-0833, Japan

${ }^{\dagger}$ Department of Engineering Informatics, Osaka Electro-Communication University, 18-8, Hatsu-cho, Neyagawa, Osaka 572-0833, Japan

Key Words:Homogeneity test, Stratified $2 \times 2$ contingency tables, Power, Monte Calro simulation
} 
best formulated in terms of relative risks rather than risk differences in view of the variable incidence of disease. In case of an equivalence trial which involves stratified $2 \times 2$ tables adjusting for a confounding variable, statistical methods have been proposed by Yanagawa et al. $(1992,1994)$ and $\operatorname{Nam}(1995)$.

In this article we propose a two-stage testing procedure that includes a preliminary test of homogeneity, corresponding to the Breslow-Day (1980) test, before demonstrating non-inferiority of a test drug in the stratified $2 \times 2$ contingency tables. Lachin \& Wei (1988) pointed out that a preliminary test of homogeneity of odds ratios over strata is needed before applying the Mantel-Haenszel (1959) test, because a heterogeneity of odds ratios often causes the Mantel-Haenszel test to reduce the power (see, Birch, 1964).

Consider the stratified $L$ pairs of independent binomial observations $\left(x_{i 11}, x_{i 01}\right)$ with denominators $\left(n_{i 1}, n_{i 0}\right)$ and success probabilities $\left(P_{i 11}, P_{i 01}\right)$ for $i=1,2, \cdots, L$. For concreteness one may think of $x_{i 11}$ and $x_{i 01}$ as the number of success among $n_{i 1}$ and $n_{i 0}$ subjects given the test drug and the control in the $i$-th stratum, respectively:

\begin{tabular}{c|cc|c}
\hline Treatment & Success & Failure & Totals \\
\hline Test drug & $x_{i 11}$ & $x_{i 12}$ & $n_{i 1}$ \\
Control & $x_{i 01}$ & $x_{i 02}$ & $n_{i 0}$ \\
\hline
\end{tabular}

For the purpose of illustration we analyzed the data from the clinical effect of neriproct suppository on internal hemorrhoids (Yanagawa et al., 1992, 1994) given in Table 1.

Table 1 Clinical Effect of Neriproct Suppository (source of data: Kinugasa et al.,1989)

\begin{tabular}{c|c|cc|c|c}
\hline Anamnesis & Treatment & Effective & Not effective & Totals & $\begin{array}{c}\text { Observed } \\
\text { odds ratio }\end{array}$ \\
\hline None & Test drug & 13 & 10 & 23 & \\
& Control & 15 & 14 & 29 & 1.21 \\
\hline Several & Test drug & 30 & 20 & 50 & \\
& Control & 27 & 18 & 45 & 1.00 \\
\hline Chronic & Test drug & 19 & 19 & 38 & \\
& Control & 8 & 23 & 31 & 2.88 \\
\hline
\end{tabular}

We consider here the null hypothesis

$$
H_{0}: P_{i 11}=f P_{i 01}, \quad i=1,2, \cdots, L
$$

against the one-sided alternative hypothesis

$$
H_{1}: P_{i 11}>f P_{i 01},
$$

where $I_{i 01}^{\prime}$ and $I_{i 11}^{\prime}$ are the efficacy rates (that is, the proportions of the patients who respond "success") for the standard (control) and the test drugs, respectively, and $f$ is a specified non-unity ratio $(0<f<1)$.

Yanagawa et al. (1992) proposed the following statistic

$$
T_{1}=\frac{\sum_{i=1}^{L}\left(x_{i 11}-n_{i 1} f \hat{P}_{i 01}\right)}{\sqrt{\sum_{i=1}^{L} \frac{n_{i 1} n_{i 0} f \hat{P}_{i 01}\left(1-f \hat{P}_{i 01}\right)^{2}}{f n_{i 1}\left(1-f \hat{P}_{i 01}\right)+n_{i 0}\left(1-f \hat{P}_{i 01}\right)}}}
$$


to test the null hypothesis (1.1) against the alternative hypothesis $(1.2)$, where $\hat{P}_{i 01}$ is the maximum likelihood estimator for $P_{i 01}$ under the null hypothesis (1.1).

If the null hypothesis $H_{0}$ is rejected at significance level $\alpha$, it is a positive evidence of non-inferiority, which is different from negative evidence of non-significance with a smaller sample size.

Let us define

$$
\varphi_{i}=\frac{P_{i 11}\left(1-f P_{i 01}\right)}{\left(1-P_{i 11}\right) f P_{i 01}}, i=1,2, \cdots, L
$$

as the odds ratio with a prescribed amount $f$ for $i$-th stratum . If we suppose that

$$
\varphi_{1}=\varphi_{2}=\cdots=\varphi_{L} \equiv \varphi
$$

then the hypothesis (1.1) and (1.2) are equivalent to

$$
H_{0}: \varphi=1 \text {, }
$$

and

$$
H_{1}: \varphi>1 \text {, }
$$

respectively. Note that when $f=1$ in eq.(1.4), the null hypothesis (1.5) reduces to that of the Breslow-Day test. In case of stratified $2 \times 2$ tables, the efficacy rates may often vary among strata (See, Rodary et al.,1989; Yanagawa et al.,1992).

\section{The test statistics}

In order to test the hypothesis (1.5), we may use the modified homogeneous uniform association model

$$
\ln \varphi_{i}=\Delta, \quad i=1,2, \cdots, L
$$

with degrees of freedom (d.f.)

$$
\text { d.f. }=L-1 \text {. }
$$

For the data given in Table 1, we may choose the response function

$$
F(\boldsymbol{P})=\left[\ln \varphi_{1}, \ln \varphi_{2}, \ln \varphi_{3}\right]^{T}
$$

as $F(\boldsymbol{P})=\boldsymbol{K} \ln \boldsymbol{A P}$ with

$$
\begin{aligned}
& \boldsymbol{P}=\left[P_{111} P_{211} P_{311} P_{112} P_{212} P_{312} P_{101} P_{201} P_{301} P_{102} P_{202} P_{302}\right]^{T} \\
& \boldsymbol{K}=\left[\begin{array}{cccccccccccc}
1 & 0 & 0 & -1 & 0 & 0 & -1 & 0 & 0 & 1 & 0 & 0 \\
0 & 1 & 0 & 0 & -1 & 0 & 0 & -1 & 0 & 0 & 1 & 0 \\
0 & 0 & 1 & 0 & 0 & -1 & 0 & 0 & -1 & 0 & 0 & 1
\end{array}\right]
\end{aligned}
$$




$$
\boldsymbol{A}=\left[\begin{array}{cccccccccccc}
1 & 0 & 0 & 0 & 0 & 0 & 0 & 0 & 0 & 0 & 0 & 0 \\
0 & 1 & 0 & 0 & 0 & 0 & 0 & 0 & 0 & 0 & 0 & 0 \\
0 & 0 & 1 & 0 & 0 & 0 & 0 & 0 & 0 & 0 & 0 & 0 \\
0 & 0 & 0 & 1 & 0 & 0 & 0 & 0 & 0 & 0 & 0 & 0 \\
0 & 0 & 0 & 0 & 1 & 0 & 0 & 0 & 0 & 0 & 0 & 0 \\
0 & 0 & 0 & 0 & 0 & 1 & 0 & 0 & 0 & 0 & 0 & 0 \\
0 & 0 & 0 & 0 & 0 & 0 & f & 0 & 0 & 0 & 0 & 0 \\
0 & 0 & 0 & 0 & 0 & 0 & 0 & f & 0 & 0 & 0 & 0 \\
0 & 0 & 0 & 0 & 0 & 0 & 0 & 0 & f & 0 & 0 & 0 \\
0 & 0 & 0 & 0 & 0 & 0 & -f & 1 & 0 & 0 & 1 & 0 \\
0 & 0 & 0 & 0 & 0 & 0 & 0 & -f & 1 & 0 & 0 & 1 \\
0 & 0 & 0 & 0 & 0 & 0 & 1 & 0 & -f & 1 & 0 & 0
\end{array}\right]
$$

where $T$ denotes the transposition of the matrix.

The response function is related to explanatory variables by the linear model

$$
F(\boldsymbol{P})=\boldsymbol{X} \boldsymbol{\xi}
$$

with

$$
\begin{aligned}
X^{T} & =[111] \\
\xi & =\Delta
\end{aligned}
$$

As noted in Gart \& Zweifel (1967), the addition of a constant $1 / 2$ to each of the cell frequencies $x_{i 11}, x_{i 12}, x_{i 01}, x_{i 02}$, provides the least biased estimates of both the Woolf's (1955) estimator and its variance. The addition of the constant $1 / 2$ will be used in this paper to estimate the logarithms of the observed within-stratum odds ratios and their asymptotic variances.

The WLS estimator $\hat{\Delta}$ of $\Delta$ may be given by

$$
\hat{\Delta}=\left(\boldsymbol{X}^{T} \boldsymbol{S}^{-1} \boldsymbol{X}\right)^{-1} \boldsymbol{X}^{T} \boldsymbol{S}^{-1} F(\boldsymbol{p})
$$

where $\boldsymbol{S}$ is the estimated variance-covariance matrix of $F(\boldsymbol{p})$ and $\boldsymbol{p}=\left[p_{111} p_{211} p_{311} p_{112} p_{212} p_{312}\right.$ $\left.p_{101} p_{201} p_{301} p_{102} p_{202} p_{302}\right]^{T}$ denotes the corresponding sample proportions to $\boldsymbol{P}$. If $f=1$ , then this estimator $\hat{\Delta}$ reduces to the modified Woolf's estimator when the odds ratios are assumed to be constant over strata (Davis,1985).

If the model (2.1) is valid, then the WLS residual statistic

$$
\mathcal{Q}_{W}=[F(\boldsymbol{p})-\boldsymbol{X} \hat{\Delta}]^{T} \boldsymbol{S}^{-1}[\boldsymbol{F}(\boldsymbol{p})-\boldsymbol{X} \hat{\Delta}]
$$

has an asymptotically $\chi^{2}$ distribution with $d . f .=L-1$. We can then test the goodnessof-fit of model (2.1), which is identical to the homogeneity of odds ratios across $L$ strata. The variance of $\hat{\Delta}$ can be estimated from

$$
\operatorname{Va} r(\hat{\Delta})=\left(\boldsymbol{X}^{T} \boldsymbol{S}^{-1} \boldsymbol{X}\right)^{-1}
$$

If the model (2.1) is valid, then we can test the null hypothesis

$$
H_{0}: \Delta=0
$$


against

$$
H_{1}: \Delta>0
$$

by the test statistic

$$
\mathcal{Q}_{c}=\frac{\hat{\Delta}}{\sqrt{\operatorname{Var} r(\hat{\Delta})}} .
$$

If the null hypothesis $(2.6)$ is true, then $\mathcal{Q}_{c}$ has an asymptotically standard normal distribution. We can reject the null hypothesis (2.6) when the corresponding $p$-value of the test statistic (2.8) is less than the significance level of $\alpha$.

Farrington \& Manning (1990) have reviewed the various methods for comparative binomial trials and recommended the test statistics based on maximum likelihood estimation. The estimators proposed in this article are asymptotically equivalent to the maximum likelihood method.

\section{Two-stage test for non-inferiority}

\subsection{Testing procedure}

The estimator $\hat{\Delta}$ has been derived from combining the within-stratum estimates, assuming the odds ratios are constant across strata, i.e., $\varphi_{1}=\varphi_{2}=\cdots=\varphi_{L} \equiv \varphi$. To illustrate why the two-stage testing proposed in the present article may be needed, consider three $2 \times 2$ tables with the population cell probabilities of $P_{i 01}=0.6, P_{i 02}=0.7, P_{i 03}=0.8$ and $P_{i 11}=1.2 P_{i 01}$. The cell probabilities may be presented as follows:

\begin{tabular}{|cc|}
\hline 0.72 & 0.28 \\
0.6 & 0.4
\end{tabular}$\quad$\begin{tabular}{cc|}
0.84 & 0.16 \\
0.7 & 0.3
\end{tabular}$\quad$\begin{tabular}{ccc}
0.96 & 0.04 \\
0.8 & 0.2 \\
\hline
\end{tabular}

It follows that

$$
\begin{aligned}
& \varphi_{1}=\frac{0.72 \times(1-0.9 \times 0.6)}{(1-0.72) \times 0.9 \times 0.6}=2.19 \\
& \varphi_{2}=\frac{0.84 \times(1-0.9 \times 0.7)}{(1-0.84) \times 0.9 \times 0.7}=3.08 \\
& \varphi_{3}=\frac{0.96 \times(1-0.9 \times 0.8)}{(1-0.96) \times 0.9 \times 0.8}=9.33
\end{aligned}
$$

The assumption of $\varphi_{1}=\varphi_{2}=\varphi_{3} \equiv \varphi$ is not valid in this situation. Even though the null hypothesis (1.5) is rejected, the estimate of the common parameter can still be interpreted as an estimate of the aggregate degree of association. In this case, analogously to the discussion due to Lachin \& Wei (1988), the test for non-inferiority may greatly reduce the power, especially when some interactions of treatments by strata exist. We must thus test the null hypothesis $H_{0}: \varphi_{1}=\varphi_{2}=\varphi_{3}$ as a preliminary test of homogeneity prior to the test for non-inferiority.

Modifying the two-stage testing procedure by Lachin \& Wei (1988) based on the WLS approach, we herein adopt the sequentially rejective algorithm: 
Step1. If the corresponding $p$-value of the test statistic (2.4) is less than $\alpha$, then the model (2.1) can not be valid and go to step 3 ; otherwise, accept the model (2.1) and continue.

Step2. If the corresponding $p$-value of the test statistic (2.8) is less than $\alpha$, then reject the null hypothesis (2.6).

Step3. After fitting the heterogeneous uniform association model (that is, full model)

$$
\ln \varphi_{i}=\Delta_{i}, \quad i=1,2, \cdots, L
$$

we can test the null hypothesis

$$
H_{0}^{(i)}: \Delta_{i}=0, \quad i=1,2, \cdots, L
$$

against

$$
H_{1}^{(i)}: \Delta_{i}>0
$$

by the test statistic

$$
\mathcal{Q}_{i}=\frac{\hat{\Delta}_{i}}{\sqrt{\operatorname{Va} r\left(\hat{\Delta}_{i}\right)}}
$$

where

$$
\hat{\Delta}_{i}=\ln \left\{\frac{p_{i 11}\left(1-f p_{i 01}\right)}{\left(1-p_{i 11}\right) f p_{i 01}}\right\}
$$

Then the significance level $\alpha_{i}$ for the test statistic $\mathcal{Q}_{i}(i=1,2, \cdots, L)$ should be set to the common value $\alpha / L$ according to the Bonferroni inequality in order to avoid multiplicity.

To illustrate how to use the proposed testing procedure, we applied it to an analysis of the data given in Table 1 . If the value $f=0.9$ was considered to be appropriate, $\mathcal{Q}_{W}=2.03(d . f .=2)$ with the corresponding $p$-value of 0.362 reveals that the model $(2.1)$ is valid. The test statistic $\mathcal{Q}_{c}=1.981$ results in a corresponding one-tail $p$-value of 0.024 .

\subsection{Examples of interactions}

Evaluation of evidence that treatment efficacy varies substantially among subsets of patients is an important feature of the analysis of clinical trials. When an interaction of treatment by subsets of patients is substantial, it is helpful to characterize the nature of the interaction. It is said that qualitative interactions occur when one treatment is superior for some subsets and the alternative treatment is superior for other subsets (Gail \& Simon,1985). A quantitative interaction arises when there is a variation in the magnitude, but not in the direction. The proposed testing allows us to evaluate the existence of any interactions of treatment by patient subset before testing non-inferiority.

To illustrate no interaction, quantitative interaction and qualitative interaction, we will consider the situation of $P_{101}=P_{201}=P_{301}=0.3 ; n_{11}=n_{10}=n_{21}=n_{20}=n_{31}=n_{30}=$ $50 ; \Gamma_{i 11}^{\prime} / P_{i 01}=1.0$.

(i) The case of no interaction

The following $2 \times 2$ tables are given and values of $\ln \varphi_{i}$ are shown in Figure 1:

\begin{tabular}{ll|}
17 & 33 \\
12 & 38 \\
\hline
\end{tabular}

\begin{tabular}{|ll|}
\hline 16 & 34 \\
14 & 36 \\
\hline
\end{tabular}

\begin{tabular}{|ll|}
\hline 20 & 30 \\
13 & 37 \\
\hline
\end{tabular}


For these tables, the two-stage testing procedure is applied:

Step1. As the value of the test statistic $(2.4)$ is $\mathcal{Q}_{W}=0.528<\mathcal{X}_{2}^{2}(0.05)$, accept the model (2.1).

Step2. As the value of the test statistic $(2.8)$ is

$$
\mathcal{Q}_{c}=\frac{\hat{\Delta}}{\sqrt{\operatorname{Va} r(\hat{\Delta})}}=2.284,
$$

we can reject the null hypothesis (2.6). Alternatively, $T_{1}=3.67$ in Yanagawa et al.'s test, and so the null hypothesis (2.6) must be rejected.

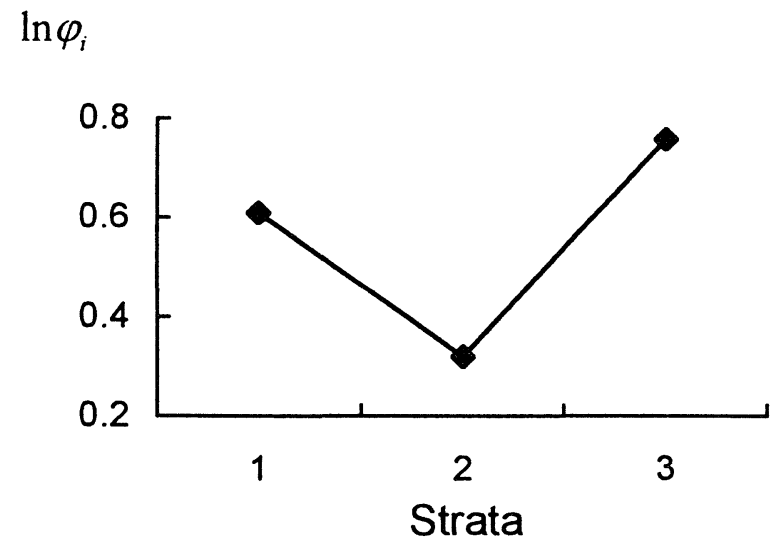

Figure 1 No interaction

(ii) The case of quantitative interaction

To illustrate the quantitative interaction occurring in the same situation, the following $2 \times 2$ tables are given and values of $\ln \varphi_{i}$ shown in Figure 2:

\begin{tabular}{|ll}
19 & 31 \\
19 & 31
\end{tabular}$\quad \quad \begin{array}{cc}23 & 27 \\
7 & 43\end{array} \quad \quad$\begin{tabular}{ll}
17 & 33 \\
18 & 32 \\
\hline
\end{tabular}

For these tables, the two-stage testing procedure is applied:

Step1. As the value of the test statistic $(2.4)$ is $\mathcal{Q}_{V V}=8.055>\chi_{2}^{2}(0.05)$, the model (2.1) can not be valid, and so go to step3.

s'tep3. We can fit the modified heterogeneous uniform association model

$$
\ln \varphi_{i}=\Delta_{i}, \quad i=1,2,3 .
$$

We can thus test the null hypothesis

$$
H I_{0}^{(i)}: \Delta_{i}=0, \quad i=1,2,3
$$

against

$$
H_{1}^{(i)}: \Delta_{i}>0
$$

As 


$$
\begin{aligned}
& \mathcal{Q}_{1}=\frac{\hat{\Delta}_{1}}{\sqrt{\operatorname{Va} r\left(\hat{\Delta}_{1}\right)}}=\frac{-0.1654}{0.4074}=-0.406, \quad \mathcal{Q}_{2}=\frac{\hat{\Delta}_{2}}{\sqrt{\operatorname{V} \hat{a} r\left(\hat{\Delta}_{2}\right)}}=\frac{1.7231}{0.4850}=3.553, \\
& \mathcal{Q}_{3}=\frac{\hat{\Delta}_{3}}{\sqrt{\operatorname{V} \hat{a} r\left(\hat{\Delta}_{3}\right)}}=\frac{0.0748}{0.4142}=0.181,
\end{aligned}
$$

we can reject the hypothesis of inferiority in the stratum 2 at the significance level of $0.05 / 3$

Alternatively, $T_{1}=2.484$ in Yanagawa et al.'s test, and so the null hypothesis $(2.6)$ must be rejected.

$$
\ln \varphi_{i}
$$

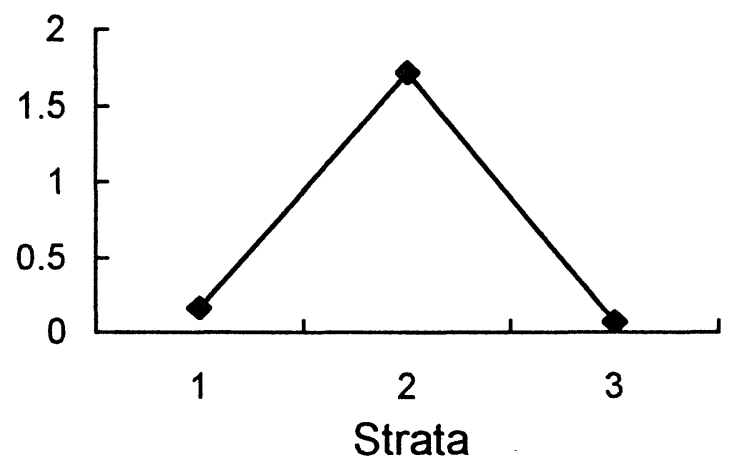

Figure 2 Quantitative interaction

(iii) The case of qualitative interaction

Consider the following $2 \times 2$ tables and values of $\ln \varphi_{i}$ shown in Figure 3:

\begin{tabular}{|cc|}
\hline 6 & 44 \\
13 & 37 \\
\hline
\end{tabular}

\begin{tabular}{|ll|}
\hline 22 & 28 \\
11 & 39 \\
\hline
\end{tabular}

\begin{tabular}{ll|}
17 & 33 \\
10 & 40 \\
\hline
\end{tabular}

For these tables, the two-stage testing procedure is applied:

Step1. As the value of the test statistic $(2.4)$ is $\mathcal{Q}_{W}=8.345>\chi_{2}^{2}(0.05)$, the model (2.1) can not be valid, and so go to step3.

stlep3. We can fit the modified heterogeneous uniform association model

$$
\ln \varphi_{i}=\Delta_{i}, \quad i=1,2,3
$$

We can thus test the null hypothesis

$$
H_{0}^{(i)}: \Delta_{i}=0, \quad i=1,2,3
$$

against

$$
H_{1}^{(i)}: \Delta_{i}>0
$$

From the test statistic (3.4), it is found that 


$$
\begin{aligned}
& \mathcal{Q}_{1}=\frac{\hat{\Delta}_{1}}{\sqrt{V \hat{a} r\left(\hat{\Delta}_{1}\right)}}=\frac{-0.7613}{0.5265}=-1.446, \quad \mathcal{Q}_{2}=\frac{\hat{\Delta}_{2}}{\sqrt{V \hat{a} r\left(\hat{\Delta}_{2}\right)}}=\frac{1.1316}{0.4379}=2.584, \\
& \mathcal{Q}_{3}=\frac{\hat{\Delta}_{3}}{\sqrt{\operatorname{Va} r\left(\hat{\Delta}_{3}\right)}}=\frac{0.8315}{0.4519}=1.828,
\end{aligned}
$$

and so we can reject the hypothesis of inferiority in the stratum 2 at the significance level of $0.05 / 3$.

Also, the statistic proposed by Yanagawa et al. (1992) is $T_{1}=2.022$ and the null hypothesis (2.6) must be rejected.

$\ln \varphi_{i}$

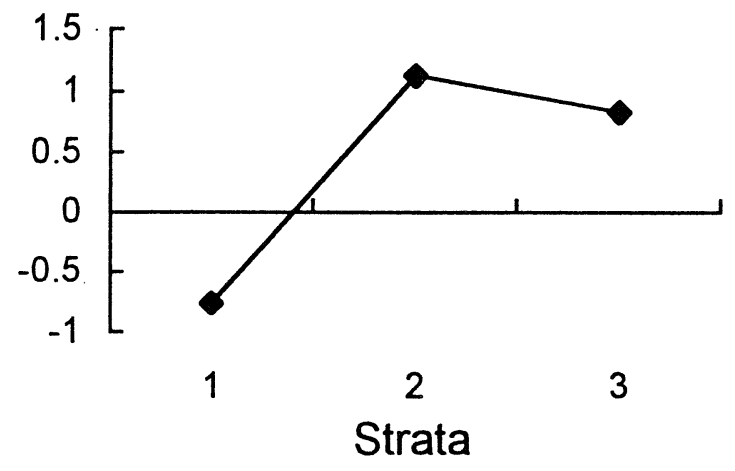

Figure 3 Qualitative interaction

\section{Monte Carlo simulation study}

\subsection{Empirical significance levels for Woolf's and Yanagawa et al.'s tests}

A Monte Carlo simulation study was conducted to assess the accuracy of the small and moderate sample properties to the null distribution and the powers of the proposed test based on the test statistic (2.8), comparing with those of other tests. The simulation study of 10000 times was designed as a $3 \times 2^{2}$ factorial with factors $P_{101}, P_{201}, P_{301}, P_{i 11} / P_{i 01}, n_{i 1}(=$ $n_{i 0}$ ) for the significance level $\alpha=0.05$ to test the null hypothesis of inferiority and $f=0.9$.

For example, setting $P_{101}=0.3, P_{201}=0.5, P_{301}=0.7, P_{i 11} / P_{i 01}=0.9$ and $f=0.9$, we obtain

$$
\begin{aligned}
& \varphi_{1}=\frac{0.27 \times(1-0.9 \times 0.3)}{(1-0.27) \times 0.9 \times 0.4}=1.0 \\
& \varphi_{2}=\frac{0.45 \times(1-0.9 \times 0.5)}{(1-0.45) \times 0.9 \times 0.5}=1.0 \\
& \varphi_{3}=\frac{0.63 \times(1-0.9 \times 0.7)}{(1-0.63) \times 0.9 \times 0.7}=1.0
\end{aligned}
$$

and the following $2 \times 2$ tables are given as the population cell probabilities: 


\begin{tabular}{|cc|}
\hline 0.27 & 0.73 \\
0.3 & 0.7 \\
\hline
\end{tabular}

\begin{tabular}{|cc|}
\hline 0.45 & 0.55 \\
0.5 & 0.5 \\
\hline
\end{tabular}

\begin{tabular}{|cc|}
\hline 0.63 & 0.37 \\
0.7 & 0.3 \\
\hline
\end{tabular}

The two-stage testing procedure is applied for various combinations $\left(n_{i 0}, n_{i 1}\right)$.

In Table 2, $T_{1}^{*}$ corresponds to $T_{1}$ in Yanagawa et al. (1992) with continuity correction and $\mathcal{Q}_{c}^{*}$ is the test statistic, which is directly calculated by the test statistic (2.8) after the addition of $1 / 2$ to each cell of each table without a preliminary test of homogeneity based on the test statistic (2.4).

Gart (1962) has shown that Woolf's estimator and maximum likelihood estimator of the odds ratio have the same asymptotic distribution, and so Woolf's estimator is asymptotically efficient.

The type I error rate of Yanagawa et al.'s test based on $T_{1}$ almost exceeds the nominal significance level, especially when the sample size for each stratum is small. Such anti-conservatism is often undesirable in clinical studies as pointed out by $\mathrm{Li}$ et al.(1979). Alternatively, $T_{1}^{*}$ and $\mathcal{Q}_{c}^{*}$ always keep the nominal significance level in these configurations, but the type I error rates of those test statistics are below $\alpha=0.05$. 
Two-Stage Testing to Establish Non-Inferiority in the Stratified $2 \times 2$ Contingency Tables

Table 2 Empirical significance level for $\alpha=0.05$

\begin{tabular}{|c|c|c|c|c|c|c|c|c|c|c|c|}
\hline$P_{101}$ & $P_{201}$ & $P_{301}$ & $n_{11}$ & $n_{10}$ & $n_{21}$ & $n_{20}$ & $n_{31}$ & $n_{30}$ & $T_{1}$ & $T_{1}^{*}$ & $\mathcal{Q}_{c}^{*}$ \\
\hline 0.3 & 0.3 & 0.3 & 10 & 10 & 10 & 10 & 10 & 10 & 0.066 & 0.034 & 0.029 \\
\hline 0.3 & 0.3 & 0.3 & 10 & 20 & 20 & 10 & 10 & 20 & 0.051 & 0.031 & 0.038 \\
\hline 0.3 & 0.3 & 0.3 & 20 & 20 & 30 & 30 & 40 & 40 & 0.048 & 0.033 & 0.043 \\
\hline 0.3 & 0.3 & 0.3 & 20 & 30 & 30 & 40 & 40 & 20 & 0.051 & 0.035 & 0.040 \\
\hline 0.3 & 0.3 & 0.3 & 30 & 30 & 30 & 30 & 30 & 30 & 0.046 & 0.032 & 0.042 \\
\hline 0.3 & 0.3 & 0.3 & 40 & 40 & 30 & 30 & 20 & 20 & 0.048 & 0.034 & 0.045 \\
\hline 0.3 & 0.3 & 0.3 & 40 & 40 & 40 & 40 & 40 & 40 & 0.058 & 0.044 & 0.043 \\
\hline 0.3 & 0.3 & 0.3 & 50 & 50 & 40 & 40 & 30 & 30 & 0.052 & 0.038 & 0.038 \\
\hline 0.3 & 0.3 & 0.3 & 50 & 50 & 50 & 50 & 50 & 50 & 0.046 & 0.035 & 0.044 \\
\hline 0.5 & 0.5 & 0.5 & 10 & 10 & 10 & 10 & 10 & 10 & 0.062 & 0.036 & 0.034 \\
\hline 0.5 & 0.5 & 0.5 & 10 & 20 & 20 & 10 & 10 & 20 & 0.052 & 0.033 & 0.036 \\
\hline 0.5 & 0.5 & 0.5 & 20 & 20 & 30 & 30 & 40 & 40 & 0.053 & 0.040 & 0.043 \\
\hline 0.5 & 0.5 & 0.5 & 20 & 30 & 30 & 40 & 40 & 20 & 0.052 & 0.038 & 0.042 \\
\hline 0.5 & 0.5 & 0.5 & 30 & 30 & 30 & 30 & 30 & 30 & 0.053 & 0.039 & 0.044 \\
\hline 0.5 & 0.5 & 0.5 & 40 & 40 & 30 & 30 & 20 & 20 & 0.052 & 0.040 & 0.044 \\
\hline 0.5 & 0.5 & 0.5 & 40 & 40 & 40 & 40 & 40 & 40 & 0.055 & 0.042 & 0.045 \\
\hline 0.5 & 0.5 & 0.5 & 50 & 50 & 40 & 40 & 30 & 30 & 0.051 & 0.040 & 0.042 \\
\hline 0.5 & 0.5 & 0.5 & 50 & 50 & 50 & 50 & 50 & 50 & 0.053 & 0.043 & 0.044 \\
\hline 0.7 & 0.7 & 0.7 & 10 & 10 & 10 & 10 & 10 & 10 & 0.056 & 0.033 & 0.029 \\
\hline 0.7 & 0.7 & 0.7 & 10 & 20 & 20 & 10 & 10 & 20 & 0.050 & 0.029 & 0.025 \\
\hline 0.7 & 0.7 & 0.7 & 20 & 20 & 30 & 30 & 40 & 40 & 0.051 & 0.037 & 0.034 \\
\hline 0.7 & 0.7 & 0.7 & 20 & 30 & 30 & 40 & 40 & 20 & 0.049 & 0.036 & 0.034 \\
\hline 0.7 & 0.7 & 0.7 & 30 & 30 & 30 & 30 & 30 & 30 & 0.053 & 0.039 & 0.036 \\
\hline 0.7 & 0.7 & 0.7 & 40 & 40 & 30 & 30 & 20 & 20 & 0.055 & 0.040 & 0.038 \\
\hline 0.7 & 0.7 & 0.7 & 40 & 40 & 40 & 40 & 40 & 40 & 0.054 & 0.041 & 0.038 \\
\hline 0.7 & 0.7 & 0.7 & 50 & 50 & 40 & 40 & 30 & 30 & 0.050 & 0.037 & 0.034 \\
\hline 0.7 & 0.7 & 0.7 & 50 & 50 & 50 & 50 & 50 & 50 & 0.053 & 0.042 & 0.039 \\
\hline 0.3 & 0.5 & 0.7 & 10 & 10 & 10 & 10 & 10 & 10 & 0.057 & 0.033 & 0.032 \\
\hline 0.3 & 0.5 & 0.7 & 10 & 20 & 20 & 10 & 10 & 20 & 0.054 & 0.033 & 0.036 \\
\hline 0.3 & 0.5 & 0.7 & 20 & 20 & 30 & 30 & 40 & 40 & 0.053 & 0.040 & 0.040 \\
\hline 0.3 & 0.5 & 0.7 & 20 & 30 & 30 & 40 & 40 & 20 & 0.051 & 0.036 & 0.043 \\
\hline 0.3 & 0.5 & 0.7 & 30 & 30 & 30 & 30 & 30 & 30 & 0.052 & 0.037 & 0.040 \\
\hline 0.3 & 0.5 & 0.7 & 40 & 40 & 30 & 30 & 20 & 20 & 0.050 & 0.036 & 0.041 \\
\hline 0.3 & 0.5 & 0.7 & 40 & 40 & 40 & 40 & 40 & 40 & 0.054 & 0.042 & 0.045 \\
\hline 0.3 & 0.5 & 0.7 & 50 & 50 & 40 & 40 & 30 & 30 & 0.050 & 0.038 & 0.041 \\
\hline 0.3 & 0.5 & 0.7 & 50 & 50 & 50 & 50 & 50 & 50 & 0.053 & 0.042 & 0.044 \\
\hline 0.6 & 0.7 & 0.8 & 10 & 10 & 10 & 10 & 10 & 10 & 0.056 & 0.033 & 0.030 \\
\hline 0.6 & 0.7 & 0.8 & 10 & 20 & 20 & 10 & 10 & 20 & 0.052 & 0.031 & 0.026 \\
\hline 0.6 & 0.7 & 0.8 & 20 & 20 & 30 & 30 & 40 & 40 & 0.051 & 0.039 & 0.035 \\
\hline 0.6 & 0.7 & 0.8 & 20 & 30 & 30 & 40 & 40 & 20 & 0.049 & 0.036 & 0.036 \\
\hline 0.6 & 0.7 & 0.8 & 30 & 30 & 30 & 30 & 30 & 30 & 0.048 & 0.037 & 0.034 \\
\hline 0.6 & 0.7 & 0.8 & 40 & 40 & 30 & 30 & 20 & 20 & 0.052 & 0.040 & 0.039 \\
\hline 0.6 & 0.7 & 0.8 & 40 & 40 & 40 & 40 & 40 & 40 & 0.052 & 0.042 & 0.038 \\
\hline 0.6 & 0.7 & 0.8 & 50 & 50 & 40 & 40 & 30 & 30 & 0.052 & 0.038 & 0.035 \\
\hline 0.6 & 0.7 & 0.8 & 50 & 50 & 50 & 50 & 50 & 50 & 0.055 & 0.042 & 0.038 \\
\hline
\end{tabular}




\subsection{Empirical significance levels and powers for two-stage testing}

In order to evaluate the behavior of the proposed two-stage testing, 10000 simulations were carried out for each combination of $P_{101}, P_{201}, P_{301}$, and sample sizes of $n_{11}, n_{10}, n_{21}, n_{20}, n_{31}, n_{30}$ in Table 2. It was desired that the significance level of the test coincides with the nominal $\alpha$ level. In Table 3, the empirical significance level of the test for $f=0.9$ (that is, the corresponding value for $P_{i 11} / P_{i 01}=0.9$ ) is closest to $\alpha=0.05$.

The empirical significance levels for the proposed two-stage testing almost maintain the nominal level in the sense that the sizes of the combined tests are never larger than the nominal size. In Table 3 , the values in the columns of $P_{i 11}=P_{i 01}$ indicate the empirical powers of deciding whether the new treatment is at least as effective as the standard treatment when they are actually equivalent.

In our simulation study, the proposed two-stage testing is less powerful than the test based on $T_{1}$. Considering the fact that most of the empirical levels for $T_{1}$ are above the nominal level, the slight reduction in power of the proposed testing may be explained by the empirical significance levels of the test being more conservative than those for $T_{1}$, which are above the nominal $\alpha$ level. Comparing the powers of the proposed testing with those of the test based on $T_{1}^{*}$, similar results are obtained for $\mathcal{Q}_{c}$ and $T_{1}^{*}$. 
Two-Stage Testing to Establish Non-Inferiority in the Stratified $2 \times 2$ Contingency Tables

Table 3. Empirical significance levels and powers

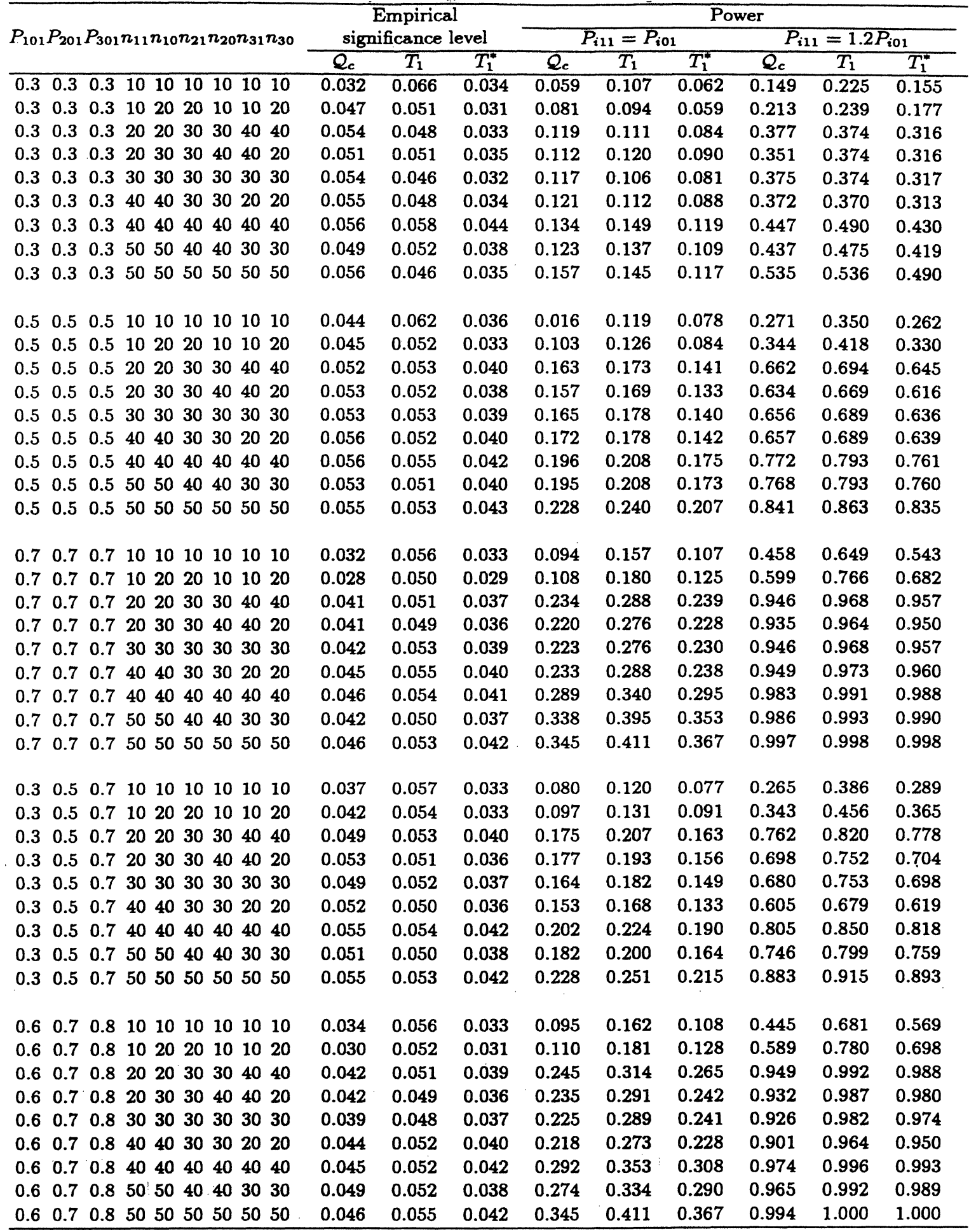




\section{KOSHIMIZU and TSUJITANI}

\section{Discussion}

The two-stage testing procedure based on the modified uniform association model is considered for demonstrating non-inferiority of a test drug to an active control. The proposed test using the WLS method is valid with moderate to large sample sizes and most powerful when conditional on the homogeneity of odds ratios being true. However, odds ratios across strata are not always homogeneous in several $2 \times 2$ tables. Then a preliminary test of homogeneity may be needed when a substantial interaction exists. In this paper, we fit the modified heterogeneous uniform association model and test the hypothesis in each stratum.

In section 3.2, we have illustrated three cases of interactions between treatment and patient subset, 1) no interaction, 2) quantitative interaction and 3) qualitative interaction. The modified uniform association model does not fit data when quantitative or qualitative interactions exist. The test statistic (2.4) allowed us to determine the presence of any interactions, while Yanagawa et al.'s test, $T_{1}$ rejected the null hypothesis of inferiority. Applying the model (3.1) with graphical display of $\ln \varphi_{i}$ may be helpful to identify the nature of the interaction.

\section{Acknowledgements}

The authors would like to thank the referees for their valuable comments and suggestions that led to substantial improvements in this presentation.

\section{REFERENCES}

Birch, M.W.(1964). The detection of partial association I: The $2 \times 2$ case. Journal of the Royal Statistical Society, B27, 111-124.

Blackwelder, W.C.(1982). "Proving the null hypothesis" in clinical trials. Controlled Clinical Trials, 3, 345-353.

Blackwelder, W.C. and Chang, M.A.(1984). Sample size graphs for "Proving the null hypothesis". Controlled Clinical Trials, 5, 97-105.

Breslow, N. and Day, N.(1980). Statistical Methods in Cancer Research:Vol.1-The Analysis of Case-Control Studies. International Agency for Research on Cancer.

Davis, L.J.(1985). Generalization of the Mantel-Haenszel estimator to nonconstant odds ratios, Biometrics, 41, 487-495.

Dunnett,C.W. and Gent, M.(1977). Significance testing to establish equivalence between treatment, with special reference to data in the form of $2 \times 2$ tables. Biometrics, 33, 593-602.

Farrington, C.P. and Manning, G.(1990). Test statistics and sample size formulae for comparative binomial trials with null hypothesis of non-zero risk difference or nonunity relative risk. Statistics in Medicine, 9, 1447-1454. 
Hirotsu, C.(1986). Some statistical problems in clinical trials (1) - Test for the equivalence of two drugs- (in Japanese). Rinshou Hyouka, 14, 467-475.

Gail,M. and Simon, R.(1985). Testing for qualitative interactions between treatment effects and patient subsets. Biometics, 41,361-372.

Gart, J.J.(1962). On the combination of relative risks, Biometrics, 18, 601-610.

Gart, J.J. and Zweifel, R.(1967). On the bias of various estimators of the logit and its variance with application to quantal bioassay, Biometrika, 54, 181-187.

Grizzle,J.E., Starmer,C.F. and Koch,G.G.(1969). Analysis of categorical data by linear Models. Biometrics, 25, 489-504.

Kinugasa, A., Hayasaka, K., Akiyama, M. et al. (1989). Clinical effect of Neriproct suppository on internal hemorrhoid: Multicenter comparative study using suppository containing Tribenoside(in Japanese). Japanese Pharmacology $\&$ Therapeutics. 17-12, 257-273.

Lachin, J.M. and Wei, L.J.(1988). Estimators and tests in the analysis of multiple nonindependent $2 \times 2$ tables with partially missing observations. Biometrics, 44, 513-528.

Li, Shou-Hua, Simon, R.M. and Gart, J.J.(1979). Small sample properties of the MantelHaenszel test. Biometrics, 66, 181-183.

Makuch, R. and Simon, R.(1978). Sample size requirements for evaluating a conservative therapy. Cancer Treatment Reports, 62, 1037-1040.

Mantel, N. and Haenszel, W.(1959). Statistical aspects of the analysis of data from retrospective studies of disease. Journal of the National Cancer Institute, 22, 719-748.

Nam, Jun-Mo(1995). Sample size determination in stratified trials to establish the equivalence of two treatments. Statistics in Medicine, 14, 2037-2049.

Rodary, C., Com-Nougue, C. and Tournade, M.(1989). How to establish equivalence between treatments: A one-sided clinical trial in paediatric oncology. Statistics in Medicine, 8,593-598.

Woolf,B.(1955). On estimating the relationship between blood group and disease. Annals of Human Genetics, 19,251-253.

Yanagawa, T., Hiejima, Y. and Sawa, J. (1992). Statistical tests for testing "Equivalence, or more than equivalence" of two drugs adjusting for prognostic factors. Japanese Journal of Biometrics, 13, 39-45.

Yanagawa, T, Tango, T. and Hiejima, Y. (1994). Mantel-Haenszel-type tests for testing equivalence or more than equivalence in comparative clinical trials. Biometrics, 50, 859-864. 